\title{
Factors Influencing the Magnitude and Clinical Significance of Drug Interactions Between Azole Antifungals and Select Immunosuppressants
}

\author{
Aline H. Saad, Pharm.D., Daryl D. DePestel, Pharm.D., and Peggy L. Carver, Pharm.D.
}

The magnitude of drug interactions between azole antifungals and immunosuppressants is drug and patient specific and depends on the potency of the azole inhibitor involved, the resulting plasma concentrations of each drug, the drug formulation, and interpatient variability. Many factors contribute to variability in the magnitude and clinical significance of drug interactions between an immunosuppressant such as cyclosporine, tacrolimus, or sirolimus and an antifungal agent such as ketoconazole, fluconazole, itraconazole, voriconazole, or posaconazole. By bringing similarities and differences among these agents and their potential interactions to clinicians' attention, they can appreciate and apply these findings in a individualized patient approach rather than follow only the one-size-fits-all dosing recommendations suggested in many tertiary references. Differences in metabolism and in the inhibitory potency of cytochrome P450 3A4 and Pglycoprotein influence the onset, magnitude, and resolution of drug interactions and their potential effect on clinical outcomes. Important issues are the route of administration and the decision to preemptively adjust dosages versus intensive monitoring with subsequent dosage adjustments. We provide recommendations for the concomitant use of these agents, including suggestions regarding contraindicated combinations, those best avoided, and those requiring close monitoring of drug dosages and plasma concentrations.

Key Words: drug interactions, azole antifungals, immunosuppressants, ketoconazole, itraconazole, fluconazole, voriconazole, cyclosporine, tacrolimus, sirolimus.

(Pharmacotherapy 2006;26(12):1730-1744)

\section{OUTLINE}

Mechanisms of Drug Interactions Mediated by

Cytochrome P450 Enzymes and Transport Proteins

Metabolism Involving the Cytochrome P450

Enzyme System

Substrates, Inducers, and Inhibitors

Genetic Polymorphism

From the Department of Pharmacy Services, University of Michigan Health System, Ann Arbor, Michigan (all authors); and the Department of Clinical Sciences, College of Pharmacy, University of Michigan, Ann Arbor, Michigan (all authors).

Address reprint requests to Peggy L. Carver, Pharm.D., College of Pharmacy, University of Michigan, 428 Church Street, Ann Arbor, MI 48109-1065; e-mail: peg@umich.edu.
Active Transport of Azoles and Immunosuppressants

Enzyme-Transporter Cooperativity

Inhibition Constant

Metabolism of Azoles

Metabolism of Cyclosporine, Tacrolimus, and Sirolimus

Drug Interactions Between Azoles and Cyclosporine Ketoconazole

Fluconazole

Itraconazole

Voriconazole

Posaconazole

Drug Interactions Between Azoles and Tacrolimus 
Ketoconazole

Fluconazole

Itraconazole

Voriconazole

Posaconazole

Drug Interactions Between Azoles and Sirolimus

Ketoconazole

Fluconazole

Voriconazole

Other Azoles

Effect of Intravenous versus Oral Administration on

Drug Interactions Between Immunosuppressants and

Azoles

Recommendations for the Clinical Management of

Drug Interactions Between Immunosuppressants and

Azoles

Additional Factors Affecting Drug Interactions

Advantages of Drug Interactions

Drug Interactions Between Azoles and Investigational

Immunosuppressants

Conclusion

The frequency of infections and mortality due to mycotic disease in the United States has increased dramatically over the past 2 decades. Among infectious disease-related deaths, those due to mycoses increased from being tenth most common in 1980 to seventh most common in 1997.' Invasive fungal infections particularly affect immunocompromised patients, including those with cancer, acquired immunodeficiency syndrome, or solid organ or bone marrow transplants, as well as certain high-risk patients with burns or critical conditions and neonates born prematurely. ${ }^{2,3}$

Fungal infections occur in $11-28 \%$ of transplant recipients. Although the highest frequency is reported in liver transplant recipients, $10-15 \%$ of patients undergoing bone marrow transplantation become infected, with a mortality rate of $60-75 \% .^{4}$ The interaction of three factors largely determine the high frequency of fungal infections in transplant recipients: technical and/or anatomic abnormalities, intensity of environmental exposure, and the patient's net state of immunosuppression. ${ }^{3}$

Fungal infections associated with immunosuppression-related, T cell-mediated defects include mucosal candidiasis, cryptococcosis, and mucormycosis, whereas neutropenia-related fungal infections include aspergillosis and disseminated candidiasis. ${ }^{4}$ These infections tend to occur early after transplantation, and their frequency decreases over time. ${ }^{5}$ In patients undergoing solid organ transplantation, the 1-6month period after the procedure is highlighted by the emergence of opportunistic infections, such as histoplasmosis, coccidioidomycosis, blastomycosis and Pneumocystis carinii (Pneumocystis jiroveci) pneumonia. ${ }^{6}$ Patients receiving bone marrow transplantation are at highest risk for fungal infections during the preengraftment period, which lasts from the day bone marrow or stem cells are infused (day 0) to approximately day 30 after transplantation. ${ }^{4}$

Given the high mortality rate associated with invasive mycoses in solid organ or bone marrow transplantation, effective prophylaxis and treatment of such infections are worthy goals. Although appropriate antifungal agents and their dosages for treating opportunistic mycoses have been defined, decisions regarding the modes of drug delivery, the patients who should receive antifungal prophylaxis, the duration of treatment or prophylaxis, and the potential for the emergence of resistant fungi remain subjects of uncertainty and controversy. ${ }^{7}$

Investigators have described the need to design a therapeutic prescription for transplant recipients that prevents and treats the two major barriers to successful transplantation: rejection and infection. The therapeutic prescription consists of an immunosuppressive program and an antimicrobial program; changes in the intensity and nature of one program obligate changes in the other. The technical and/or anatomic consequences of transplantation, the time course of infection after transplantation, and the possibility of drug interactions with the immunosuppressive program greatly influence the use of antimicrobials in transplant recipients. ${ }^{8}$

Drug interactions between immunosuppressants and azoles are discussed elsewhere. ${ }^{9-12}$ Our objective was to describe certain studies to illustrate similarities and differences among these interactions so that clinicians can appreciate and apply these findings in an individualized patient approach rather than follow only the one-sizefits-all dosing recommendations suggested in many tertiary references. Clinicians must be aware of the combined influence of drug transport and metabolic routes on the likelihood and magnitude of drug interactions. Because data are not available for all potential drug-drug combinations, a thorough understanding of the principles underlying drug interactions is crucial to anticipate potential interactions with new or existing agents used in the clinical setting. 


\section{Mechanisms of Drug Interactions Mediated by Cytochrome P450 Enzymes and Transport Proteins}

Metabolism Involving the Cytochrome P450 Enzyme System

A thorough understanding of the mechanisms of drug interactions provides clinicians the ability to predict such interactions and to devise strategies to minimize or avoid those of greatest clinical significance. ${ }^{13}$ Drug interactions can be categorized as pharmacodynamic or pharmacokinetic. Pharmacodynamic interactions include additive, synergistic, or antagonistic interactions that can affect efficacy or toxicity. Pharmacokinetic interactions result when one drug alters the absorption, distribution, metabolism, or excretion of another drug.

Inhibition or induction of hepatic and extrahepatic cytochrome P450 (CYP) enzymes is the most common mechanism of drug interactions. ${ }^{13}$ The major site of drug metabolism is the liver, where two types of reactions occur. In phase I reactions catalyzed by CYP enzymes, oxidation of a parent drug yields a more polar, hydrophilic moiety that may be pharmacologically active or inactive. In phase II reactions, which CYP enzymes do not mediate, conjugation of the parent drug or previously oxidized drug yields a hydrophilic moiety that is excreted in the feces or urine more readily than the parent drug. ${ }^{14}$

Cytochrome P450 enzymes are heme proteins that catalyze phase I metabolism of many endogenous substrates, including steroids, fatty acids, prostaglandins, bile acids, and xenobiotics (including drugs, carcinogens, environmental pollutants, and many synthetic chemicals). ${ }^{14}$ These enzymes are found in the smooth endoplasmic reticulum of liver hepatocytes and in the villous columnar epithelium of the jejunum, lungs, kidney, and brain. More than 40 CYP enzymes have been identified in humans. Of these, CYP3A4, CYP2D6, CYP1A2, CYP2C9, CYP2C19, and CYP2E1 are responsible for metabolizing nearly all clinically useful drugs. ${ }^{15}$

The observation that CYP3A4 metabolizes nearly $50 \%$ of all clinically used drugs and endogenous steroids may explain why CYP3A4, among the CYP enzymes, is most often involved in drug metabolism and drug interactions. ${ }^{16}$ This enzyme accounts for $29 \%$ and $70 \%$ of the total human hepatic and gastrointestinal tract CYP enzymes, respectively, affecting both presystemic and systemic drug distribution. ${ }^{14,17}$ This first- pass elimination results in variable (often low) oral bioavailability of CYP3A4-metabolized drugs.

An important characteristic of CYP3A is its large interindividual variability in activity, which reflects a genetic effect combined with modulation by environmental factors. The inhibition and induction of enzymes often substantially increases this variability. Hepatic CYP3A4 varies at least 20-fold, and enteric CYP3A4 varies 10fold among individuals. However, the CYP3A4 content of each site appears to be regulated independently. Thus, one patient might have high CYP3A4 activity in the liver and low CYP3A4 activity in the intestine, whereas another patient has the opposite activities; the liver is the major site of drug metabolism in the first patient, whereas the intestine is the major site in the second. ${ }^{18}$ Relative contributions of hepatic and enteric metabolism can be estimated separately by determining differences in drug clearance after oral and intravenous administration of a CYP3A4-metabolized drug. ${ }^{17}$

The CYP group of enzymes can be highly substrate specific and therefore capable of metabolizing only a few substrates, or they may be poorly specific and capable of metabolizing a broad range of substrates. The degree of enzyme specificity greatly affects the outcome of its inhibition. The broader the enzyme specificity, the more drug interactions its inhibition is likely to elicit. To predict, evaluate, and manage such drug interactions, clinicians must first identify the enzymes responsible for each agent's metabolism. Then, they must consider the dosages and timing of administration of the drugs in question, the duration of therapy, the baseline and steady state concentrations, the therapeutic index of each agent, and the potential for interindividual variability in pharmacokinetic variables, including absorption, distribution, metabolism, and elimination. ${ }^{13}$

\section{Substrates, Inducers, and Inhibitors}

Drugs can be substrates, inducers, and inhibitors of CYP enzymes. Substrates are moieties that undergo metabolism by one or more CYP enzymes. Inducers increase the amount or activity of a CYP enzyme. By contrast, inhibitors decrease the amount or activity of the enzyme. Enzyme inducers and inhibitors produce their effects in a dose- and concentration-dependent manner; within a range, high doses or concentrations of drugs generally increase induction or 
inhibition. Inducers and inhibitors differ in the onset and duration of their effects on CYP enzymes. Although maximum enzyme induction is generally achieved over approximately 2 weeks, enzyme inhibition can be observed immediately after the administration of the first dose of an inhibitor drug. ${ }^{14-16}$ Limited data are available regarding the duration of enzyme inhibition or induction after inducers or inhibitors are discontinued; however, the halflives and protein binding of inducers and inhibitors are factors.

Enzyme induction refers to an increase in enzymatic activity due to increased production of the enzyme (by means of enhanced transcription and translation) or due to a reduction in the natural rate of its breakdown. The CYP enzymes that are known to be inducible are CYP1A2, CYP2C9, CYP2E1, and CYP3A4. ${ }^{19}$ Unlike CYP inhibitors, which have an immediate effect, enzyme induction is gradual. Once an enzyme is induced, removal of the inducing agent eventually results in normalization of enzyme activity. Common inducers are phenytoin, carbamazepine, rifampin, and ethanol. As can be predicted, these agents often increase the metabolism of the other CYP substrates, potentially resulting in therapeutic failures. ${ }^{20,21}$

In general, for CYP3A4 substrates (e.g., immunosuppressants) with low oral bioavailability (high presystemic or first-pass elimination), administration of a single dose of a CYP3A4 inhibitor substantially increases the area under the plasma concentration-time curve (AUC) for the substrate. By contrast, concomitant administration of a single dose of a CYP3A4 inhibitor with a CYP3A4 substrate with high oral bioavailability does not produce a large effect. However, repeat administration of both may cumulatively increase plasma substrate concentrations, and a clinically significant interaction may occur only during steady-state conditions. ${ }^{17}$

An inhibitor can be classified as competitive or noncompetitive. By mimicking the substrate, a competitive inhibitor competes for binding to the active site of an enzyme. For a competitive inhibitor to fully inhibit a site, high concentrations are needed. The higher the concentration, the stronger the inhibition. The clinical significance of drug interactions resulting from competitive inhibition depends on the concentration of the inhibitor achieved at the site of inhibition, the relative doses of the inhibitor and the substrate, the relative bioavailability, the relative affinity constants of the inhibitor and substrate (i.e., how tightly they bind to the site), the interindividual variability, and the therapeutic indices of the drugs. ${ }^{9}$ At equimolar concentrations, ketoconazole is the most potent CYP3A4 inhibitor among the azoles, followed by itraconazole, voriconazole, and fluconazole.

A noncompetitive inhibitor binds to a location on the enzyme other than the active site, altering its conformation so that the active site is no longer fully functional. Only minimal amounts of a noncompetitive inhibitor are needed for inhibition to be effective. Table 1 summarizes the metabolism of azoles, the CYP enzymes involved, and the type and relative potency of inhibition. ${ }^{22-25}$

\section{Genetic Polymorphism}

A major cause of drug metabolism and subsequent drug effects is genetic polymorphism, in particular CYP2D6, CYP2C9, and CYP2C19. The distribution of these enzymes in the population is polymodal, as determined by genetic polymorphism. Although CYP3A4 drugmetabolizing activity varies widely among individuals, notable polymorphisms have not been identified. ${ }^{17}$

\section{Active Transport of Azoles and Immunosuppressants}

In addition to CYP enzymes, active transporters (e.g., P-glycoprotein [P-gp]), and the organic anion-transporting polypeptides (e.g., hepatic canalicular efflux transporter MRP2) play an important role in drug interactions. Active drug transporters play a key role in regulating access of drugs to the drug-metabolizing enzymes and controlling drug concentrations in enterocytes and hepatocytes.

A plasma membrane-associated glycoprotein, $\mathrm{P}$-gp is a member of the adenosine $5^{\prime}$ - triphosphate (ATP)-binding cassette transporter superfamily and a product of the multidrug resistance 1 (MDR1) gene. Although variably expressed in the population, P-gp is present at high levels in the liver, kidney, pancreas, small intestine, colon, and adrenal gland, as well as in the capillary endothelium of the brain and testes. This glycoprotein functions as an ATP-dependent efflux pump that excretes xenobiotics into bile, gastrointestinal tract, and urine and that prevents access to the central nervous system by limiting transport across the blood-brain barrier. These actions lower plasma and cerebrospinal fluid concentrations of xenobiotics, suggesting that P- 
Table 1. Metabolism of Azoles and Immunosuppressants ${ }^{22-25}$

\begin{tabular}{|c|c|c|c|c|c|c|}
\hline \multirow[b]{2}{*}{ Agent } & \multicolumn{2}{|c|}{$\mathrm{CYP}^{\mathrm{a}}$} & \multicolumn{2}{|c|}{ Inhibition of CYP3A4 } & \multicolumn{2}{|c|}{ P-glycoprotein } \\
\hline & Substrate & Inhibitor & Potency & Type & Substrate & Inhibitor \\
\hline$\overline{\text { Ketoconazole }}$ & $3 \mathrm{~A} 4$ & $3 \mathrm{~A} 4,2 \mathrm{C} 19$ & ++++ & $\begin{array}{l}\text { Competitive, } \\
\text { noncompetitive }\end{array}$ & Yes & Yes \\
\hline Fluconazole & $3 \mathrm{~A} 4$ & $2 \mathrm{C} 9,2 \mathrm{C} 19$ & + & $\begin{array}{l}\text { Noncompetitive, } \\
\text { mixed }\end{array}$ & Yes & No \\
\hline Itraconazole & $3 \mathrm{~A} 4$ & $3 \mathrm{~A} 4$ & +++ & Competitive & Yes & Yes \\
\hline Hydroxyitraconazole & $3 \mathrm{~A} 4 ?$ & $3 \mathrm{~A} 4$ & ND & Competitive & ND & ND \\
\hline Ketoitraconazole & $3 \mathrm{~A} 4$ & $3 \mathrm{~A} 4$ & ND & ND & ND & ND \\
\hline N-desalkylitraconazole & $3 \mathrm{~A} 4$ & $3 \mathrm{~A} 4$ & ND & ND & ND & ND \\
\hline Voriconazole & $3 \mathrm{~A} 4$ & $\begin{array}{l}3 \mathrm{~A} 4,2 \mathrm{C} 9 \\
2 \mathrm{C} 19\end{array}$ & ++ to +++ & $\begin{array}{l}\text { Competitive, } \\
\text { noncompetitive }\end{array}$ & ND & ND \\
\hline Posaconazole & $\mathrm{ND}^{\mathrm{b}}$ & $3 \mathrm{~A} 4$ & $+++^{c}$ & ND & Yes & Yes \\
\hline Cyclosporine & $3 \mathrm{~A} 4$ & $3 \mathrm{~A} 4$ & ND & Competitive & Yes & Yes \\
\hline Tacrolimus & $3 \mathrm{~A} 4$ & ND & ND & ND & Yes & Yes \\
\hline 13-O-desmethyltacrolimus & ND & ND & ND & ND & Yes & Yes \\
\hline Sirolimus & $3 \mathrm{~A} 4$ & ND & ND & Competitive & Yes & Yes \\
\hline Everolimus & $3 \mathrm{~A} 4$ & ND & ND & ND & Yes & Yes \\
\hline
\end{tabular}

$\mathrm{CYP}=$ cytochrome $\mathrm{P} 450 ; \mathrm{ND}=$ no data.

${ }^{a}$ CYP enzyme involved in the metabolism of the azole.

${ }^{\mathrm{b}}$ To date, none have been identified; does not inhibit CYP3A4

'Based on limited data.

gp has a role in defense against xenobiotics. ${ }^{15}$ In the gastrointestinal tract, P-gp is located in the brush border on the apical (luminal) surface of mature enterocytes. The colon has the highest Pgp expression, and the stomach and jejunumileum, the lowest. ${ }^{26}$

\section{Enzyme-Transporter Cooperativity}

Both P-gp and CYP3A4 share some substrate overlap and are colocalized in the small intestine, where they form a cooperative barrier that limits the oral bioavailability of xenobiotics and drugs, such as immunosuppressants. Substrates (e.g., immunosuppressants) for P-gp and CYP3A4 entering an enterocyte may be absorbed directly into the systemic circulation, metabolized by CYP3A4 in the enterocyte, or secreted back into the intestinal lumen by P-gp. Drug pumped back into the lumen may be reabsorbed at a distal site and exposed again to any of the three fates just described. ${ }^{18}$

For dual-substrate drugs, repeated pumping out of the enterocyte by P-gp limits and regulates their access to CYP3A4 metabolism and prevents high drug concentrations in the enterocyte from overwhelming the enzyme. Therefore, the two proteins function in concert to reduce the intracellular concentration of xenobiotics. ${ }^{22,27}$

Coordinate upregulation of CYP3A was demonstrated in a cell culture model in response to some xenobiotics. However, other drugs have caused selective upregulation of P-gp expression. ${ }^{12}$ Despite this shared functionality, no correlation was found between intrasubject enterocyte P-gp levels and CYP3A4 in the small intestine and/or liver. Moreover, CYP3A4 and P-gp do not appear to be coordinately regulated. ${ }^{28}$

The uptake of P-gp substrates can become saturated. When this occurs or when P-gp is inhibited, passive diffusion becomes the ratelimiting step in absorption. Therefore, increased doses of cyclosporine can increase the rate and extent of drug absorption. Drugs with high partition coefficients (i.e., highly lipophilic agents) can diffuse rapidly. The azoles, which are P-gp substrates, differ in their lipophilicity. Inhibition or saturation of P-gp has greatest effect on the oral bioavailability of water-soluble agents (e.g., fluconazole) because relatively lipophilic agents (e.g., itraconazole) can rapidly diffuse across the enterocyte.

\section{Inhibition Constant}

Clinical observations suggest that azoles have various potencies with respect to their ability to inhibit CYP3A4 and P-gp. ${ }^{29}$ Determining the in vitro inhibition constant, $K_{i}$, which quantifies the molecular interaction and inhibitory potency of an agent, assists in predicting the magnitude of in vivo drug interactions. For a specific enzyme, $\mathrm{K}_{\mathrm{i}}$ is a constant that is largely independent of substrate identity. This constant can be used to 
predict enzyme behavior over a wide range of substrate and enzyme concentrations and, theoretically, to compare the inhibition potency of different drugs. ${ }^{30}$ As $\mathrm{K}_{\mathrm{i}}$ decreases, the potency of the inhibitor increases exponentially.

The most potent reversible CYP3A inhibitors, which include azole antifungal agents and human immunodeficiency virus protease inhibitors, have $\mathrm{K}_{\mathrm{i}}$ values below $1 \mu \mathrm{mol}$. Clinically significant inhibition is uncommon for compounds with values above $75-100 \mu \mathrm{mol}$ because sufficiently high concentrations are not clinically achieved. ${ }^{31}$, 32 For example, ketoconazole and fluconazole are inhibitors of human hepatic and intestinal CYP3A4. However, ketoconazole is a more potent inhibitor than fluconazole, with mean \pm SD $\mathrm{K}_{\mathrm{i}}$ values of $14.9 \pm 6.7$ and $17.0 \pm 7.9 \mathrm{nmol}$ for hepatic and intestinal microsomes, respectively; by contrast, $\mathrm{K}_{\mathrm{i}}$ values for fluconazole are $10.7 \pm 4.2$ and $10.4 \pm 2.9 \mu \mathrm{mol}$, respectively. These data reflect a 1000 -fold difference in the magnitude of CYP3A4 inhibition. ${ }^{33}$ An extensive review on the use of inhibitory constants to evaluate the potential for drug-drug interactions has been published. ${ }^{34}$

The practice of using in vitro-in vivo scaling procedures to predict the effect of the coadministration of CYP inhibitory agents on in vivo drug interactions has many limitations. First, because in vitro studies are performed in human or animal preparations of liver microsomes, they do not reflect the possible contributions of extrahepatic CYPs and P-gp. ${ }^{17}$ Second, concentrations of the inhibitor at the active site of the enzyme in vitro may not mirror concentrations in vivo. ${ }^{35}$ Third, in vitro experiments are usually conducted in media that are devoid of plasma proteins and that do not mimic the in vivo setting with respect to $\mathrm{pH}$, ionic strength, or protein binding, all of which can affect enzyme activity. ${ }^{30}$ Finally, metabolites of the inhibitor may contribute to overall inhibitory effects on the enzyme. In general, the overall limitation is that a given concentration of an inhibitor is more potent in vivo than it is in vitro. ${ }^{35}$ As a result, human data should be obtained whenever possible. ${ }^{17}$ However, in vitro drug interactions can be used as screening tools that provide the rationale for further, in-depth examination of the interaction in human clinical trials.

Metabolism of Azoles

Currently available azoles inhibit the fungal
CYP3A4-dependent enzyme lanosterol 14- $\alpha$ demethylase. They hinder the synthesis of ergosterol, the major sterol component of fungal plasma membranes, resulting in altered membrane fluidity and inhibition of fungal growth and replication. ${ }^{36}$ Early azoles, such as ketoconazole, are poorly selective in the inhibition of fungal versus mammalian CYP3A4. As a result, they can cross-inhibit mammalian CYP3A4, leading to drug interactions with other CYP3A4-metabolized drugs and to adverse effects (e.g., gynecomastia, adrenocortical insufficiency) due to the inhibition of CYP3A4-mediated metabolism of human steroid hormones. ${ }^{36,37}$ New azoles have enhanced selectivity for fungal versus mammalian CYP3A4. As a consequence, they have improved toxicity and drug-interaction profiles. $^{36}$

The lipophilicity and lack of aqueous solubility of azoles influence the likelihood of their interacting with immunosuppressants. Ketoconazole, itraconazole, voriconazole, and posaconazole are more lipophilic than fluconazole and require extensive oxidative CYP metabolism to hydrophilic metabolites in order to be eliminated from the body. ${ }^{36,38,39}$ Itraconazole, the most lipophilic and water-insoluble azole, is prone to enzymatic and transporter-mediated interactions in the intestine, liver, and kidney that interfere with the absorption and elimination of it and other drugs.

More than 30 metabolites have been proposed for itraconazole, only one of which, hydroxyitraconazole, has been studied in humans. The biotransformation of itraconazole is complex and incompletely understood; however, CYP3A4 catalyzes most, if not all, of its metabolism. In addition to hydroxyitraconazole, which is formed primarily in the intestine by CYP3A4, itraconazole and hydroxyitraconazole are converted to ketoitraconazole. In addition, CYP3A4 catalyzes the conversion of ketoitraconazole, and possibly itraconazole and hydroxyitraconazole, to $\mathrm{N}$ desalkylitraconazole. Hydroxyitraconazole possesses antifungal activity similar to that of itraconazole, but it circulates at increased plasma concentrations. Itraconazole is a relatively potent inhibitor of CYP3A4 in vitro. Recent in vitro studies demonstrated that hydroxyitraconazole, ketoitraconazole, and N-desalkylitraconazole are CYP3A4 inhibitors at least as potent as itraconazole; therefore, they may contribute substantially to the inhibition of CYP3A4 observed clinically. The long half-life of itraconazole and its pharmacologically active 
metabolites prolong the inhibitory effects on CYP3A4 metabolism of immunosuppressants. ${ }^{38,39}$

Voriconazole, which is lipophilic and which has limited water solubility, is well absorbed orally and less prone than other drugs to presystemic interactions in the intestine. Voriconazole drug interactions are dose dependent. Because it has unpredictable nonlinear pharmacokinetics, its drug interactions are difficult to predict and manage. The hepatic biotransformation of voriconazole is fairly complex and involves CYP2C19, CYP3A4, and CYP2C9. The principle $\mathrm{N}$-oxide metabolite of voriconazole is formed by CYP2C19 and CYP3A4 and, to some extent, CYP2C9. ${ }^{38,39}$ Two CYPs involved in voriconazole metabolism, CYP2C19 and CYP2C9, exhibit genetic polymorphism. Variability in the CYP2C19 genotype accounts for approximately $30 \%$ of the overall between-subject variability in voriconazole pharmacokinetics. ${ }^{40}$

Homozygous poor metabolizers have the highest plasma concentrations of voriconazole, followed by heterozygous extensive metabolizers, then homozygous extensive metabolizers. After oral and intravenous dosing with $200 \mathrm{mg}$ and 3 $\mathrm{mg} / \mathrm{kg}$ every 12 hours, respectively, mean AUCs in poor metabolizers and heterozygous extensive metabolizers were approximately 4- and 2-fold higher, respectively, than those of extensive metabolizers. The high blood concentrations of voriconazole observed in poor metabolizers may increase the magnitude of their drug interactions; close monitoring of plasma concentrations may be required in these individuals. ${ }^{41}$ Furthermore, CYP2C9 expresses polymorphism, which, if expressed, is associated with reduced enzymatic activity. The polymorphism is most prevalent among Caucasians, less frequent among AfricanAmericans, and absent in Asians. The magnitude of interactions with CYP3A4 substrates varies, ranging from no interaction (indinavir) to large increases in exposure (sirolimus).

By contrast, fluconazole is only slightly lipophilic and highly water soluble. It is well absorbed orally and less prone to drug interactions than other drugs, particularly in the intestine. ${ }^{38,39}$ Drugs that interact with fluconazole or voriconazole are substrates of CYP2C9, CYP2C19, or CYP3A4. The CYPmediated interactions with fluconazole are often dose dependent. Because fluconazole has linear and predictable pharmacokinetics, these interactions may be avoided or minimized by using the lowest effective dose.

Posaconazole, an azole recently approved by the U.S. Food and Drug Administration, is active against a broad spectrum of fungi, including Aspergillus and Candida species and Zygomycetes. In vitro studies demonstrated that posaconazole is an inhibitor but not a substrate of hepatic (but not total) CYP3A $4^{25}$ and that it is both a substrate and an inhibitor of P-gp. This observation suggests that it may have a drug-interaction profile similar to that of other azoles. In addition, posaconazole undergoes glucuronidation by uridine $5^{\prime}$-diphosphate-glucuronosyltransferase enzymes. ${ }^{42}$

All five azoles inhibit CYP3A4 but with various potencies. Ketoconazole is the most potent inhibitor, followed by itraconazole and voriconazole (roughly equipotent), and then fluconazole (Table 1). ${ }^{38,41,43,44}$ The relative inhibitory potency of posaconazole is not well described at this time. Its in vitro $\mathrm{K}_{\mathrm{i}}$ values have not been published, and the few clinical trials of CYP3A4 substrates have been reported mainly in abstract form. ${ }^{24,25,45}$ Differences in relative potency translate into various degrees of interaction when each of the azoles is combined with a CYP3A4 substrate (as discussed later).

In clinical practice, fluconazole is the most commonly used agent followed by voriconazole, itraconazole, and, finally, ketoconazole. Although new azoles have widely replaced ketoconazole, its drug interactions with immunosuppressants are important because it is the most potent inhibitor and because it represents an important model for drug-drug interactions.

Metabolism of Cyclosporine, Tacrolimus, and Sirolimus

Both intestinal and hepatic CYP3A4 metabolize all immunosuppressants. Cyclosporine undergoes substantial presystemic metabolism; its oral bioavailability ranges from $30-70 \%$. Tacrolimus is available as oral and parenteral formulations. Its oral absorption is incomplete and variable, with an oral bioavailability of $10-30 \% .{ }^{46}$ Sirolimus is available as an oral solution and tablets, both of which have wide interpatient and intrapatient variability in oral absorption. Although the oral bioavailability of sirolimus is not known precisely, it has been estimated to be about $15 \% .{ }^{47}$

Cyclosporine, tacrolimus, and sirolimus are substrates of CYP3A4 and both substrates and inhibitors of P-gp. As a consequence, they have an immense potential for clinically significant drug-drug interactions that result in increased 
plasma concentrations of immunosuppressants, excessive immunosuppression, and toxicity. Because more than $50 \%$ of the hepatic metabolism of cyclosporine, tacrolimus, or sirolimus involves CYP3A4, drug interactions resulting in complete induction or inhibition of CYP3A4 are expected to lead to clinically significant pharmacokinetic drug interactions. ${ }^{28}$

Because the correlation between dose and plasma concentrations is poor, with wide variability in interindividual and intraindividual pharmacokinetics, and because many of the adverse effects of immunosuppressants (especially nephrotoxicity) are dose and plasma concentration related, close monitoring of plasma concentrations is necessary to guide dosage adjustments, to minimize dose-related toxicity, and to maximize efficacy. ${ }^{17,28,46-48}$

In clinically administered dosages, cyclosporine appears to exert an inhibitory effect on P-gp more potent than that of tacrolimus or sirolimus. ${ }^{26}$ Although nephrotoxicity is a wellrecognized, concentration-dependent adverse effect of cyclosporine and tacrolimus, the relationship between plasma concentrations and neurotoxicity is hypothetical. Some have proposed that cyclosporine- or tacrolimusinduced inhibition of P-gp in the brain facilitates the distribution of immunosuppressants in the central nervous system, increasing concentrations of immunosuppressants in brain tissue. ${ }^{28}$ However, plasma concentrations of sirolimus have not been correlated with any of its documented adverse events. ${ }^{47}$

\section{Drug Interactions Between Azoles and Cyclosporine}

Key steps in the metabolism of immunosuppressants are accomplished by CYP3A4. Azoles inhibit this enzyme, decreasing metabolism, increasing serum concentrations of immunosuppressants, and raising the potential for immunosuppressant-induced toxicity (particularly renal), overimmunosuppression, and opportunistic infections. ${ }^{8}$ Yet, the degree of inhibition of CYP3A4 by individual azoles varies, and drug interactions with immunosuppressants are best described as an agent-specific rather than class effect for all agents in the class.

\section{Ketoconazole}

Among drug interactions between immunosuppressants and azoles, the interaction between cyclosporine and ketoconazole is the best studied. As the most potent CYP3A4 inhibitor of the azoles and an inhibitor of P-gp, ketoconazole and its concomitant administration with cyclosporine increases the AUC of cyclosporine almost 3-fold, allowing for cyclosporine dosage reductions of $70-80 \%{ }^{49}$ The magnitude of this interaction becomes fully apparent days to weeks after the addition of ketoconazole to cyclosporine. The interaction resolves $7-10$ days after ketoconazole is discontinued. ${ }^{50}$

\section{Fluconazole}

Fluconazole is a less potent inhibitor of CYP3A4 than ketoconazole, itraconazole, or voriconazole, and it is not an inhibitor of P-gp. ${ }^{12}$ Fluconazole inhibition of CYP3A4 appears to be dose dependent, and interactions with cyclosporine are generally important only with doses of at least $200 \mathrm{mg} /$ day. Although early reports of fluconazole and cyclosporine coadministration suggested a lack of interaction, later reports noted that administration of oral fluconazole 200 $\mathrm{mg}$ /day for 14 days doubled the AUC for cyclosporine. ${ }^{51}$ Whether fluconazole 800-1200 $\mathrm{mg} /$ day, which is often used to treat invasive candidal infections, further increases the AUC is unknown.

\section{Itraconazole}

Itraconazole inhibits intestinal and hepatic CYP3A4-mediated metabolism of cyclosporine. Its effect on cyclosporine pharmacokinetics varies. Some, but not all, patients had elevated cyclosporine concentrations during concomitant administration of itraconazole. ${ }^{50}$ Itraconazole generally doubles cyclosporine trough concentrations. Inhibitory effects of itraconazole on CYP3A4 metabolism of immunosuppressants persist for several weeks after itraconazole is discontinued because of its long half-life and its pharmacologically active metabolites. ${ }^{38,39}$

\section{Voriconazole}

Data regarding the interaction between voriconazole and cyclosporine are somewhat limited. In kidney transplant recipients whose condition was stabilized for at least 4 weeks with individualized regimens of twice-daily cyclosporine, the addition of oral voriconazole $200 \mathrm{mg}$ twice/day to oral cyclosporine 150-375 mg/day increased the mean AUC for cyclosporine 1.7fold. Because of these findings, the authors suggested that the daily dose of oral cyclosporine 
be decreased $50 \%$ when voriconazole therapy is started. ${ }^{40,52}$ However, in patients who withdrew from the study, trough concentrations of cyclosporine increased as much as 3 -fold..$^{52}$

\section{Posaconazole}

In four heart transplant recipients, the dose of cyclosporine was reduced $0-29 \%$ after the addition of oral posaconazole $200 \mathrm{mg}$ twice/day for 10 days. This outcome suggested that low doses of posaconazole, similar to fluconazole, did not substantially inhibit CYP3A4. ${ }^{25}$

\section{Drug Interactions Between Azoles and Tacrolimus}

Drug interactions involving tacrolimus are less well characterized in terms of their mechanism, onset, potency, and clinical relevance than those with cyclosporine. The in vitro data are derived from studies performed in various species (rat, human, pig) and tissues (liver, small intestine, gastrointestinal mucosa). All demonstrated differing magnitudes of azole inhibition of CYP3A4-mediated metabolism of tacrolimus in the liver and gastrointestinal tract. ${ }^{50}$ Available in vitro data are poorly predictive of clinical results in humans, and few controlled drug trials have addressed this issue. ${ }^{28}$

\section{Ketoconazole}

The addition of oral ketoconazole $200 \mathrm{mg}$ /day to the regimen of a patient whose condition was stabilized with tacrolimus prompted a reduction in tacrolimus doses by as much as $80 \% .^{53}$ The interaction was observed within 1 day after the oral ketoconazole was started and persisted for about 7 days after it was discontinued. Despite a preemptive decrease in the tacrolimus dosage by $45 \%$ when ketoconazole was begun, tacrolimus plasma concentrations became supratherapeutic.

\section{Fluconazole}

Researchers investigated the interaction between low-dose oral fluconazole $100 \mathrm{mg} /$ day for 7 days and oral tacrolimus in a prospective randomized study of 19 renal allograft recipients. $^{54}$ Within 5 days of the start of fluconazole, doses of tacrolimus were decreased substantially. The investigators concluded that a tacrolimus dose equivalent to $60 \%$ of the initial dose (i.e., a $40 \%$ reduction in the dose of tacrolimus) was appropriate during coadministration with fluconazole. Further dose reductions of tacrolimus are presumably needed with doses of fluconazole higher than this; however, this possibility has not been evaluated. Other researchers reported that, after fluconazole was discontinued, 9 days were required for evidence of the drug interaction to resolve. ${ }^{55}$

\section{Itraconazole}

Specific data regarding the interaction between itraconazole and tacrolimus are limited to retrospective studies and case reports. Several investigators demonstrated that, with the addition of oral itraconazole $200-400 \mathrm{mg}$ /day to tacrolimus, a 50-66\% decrease in the dose of tacrolimus was needed. ${ }^{56-58}$ The long half-life of itraconazole prolonged the inhibition of tacrolimus metabolism. A report of a kidney transplant recipient described an interaction between oral itraconazole $100 \mathrm{mg}$ twice/day and tacrolimus. ${ }^{56}$ The onset of the interaction was apparent less than 2 days after the start of itraconazole. Despite the discontinuation of itraconazole after 5 days of therapy, the interaction persisted for an additional 7 days, and a $50 \%$ reduction in the dose of tacrolimus was required. Itraconazole $600 \mathrm{mg}$ given orally twice/day increased tacrolimus trough concentrations nearly 5-fold, highlighting the dosedependent inhibition and nonlinear pharmacokinetics observed with this agent. ${ }^{57}$

\section{Voriconazole}

Among healthy volunteers who had received oral voriconazole $400 \mathrm{mg}$ twice/day for 1 day followed by $200 \mathrm{mg}$ twice/day for 6 days, a single oral dose of tacrolimus $0.1 \mathrm{mg} / \mathrm{kg}$ tripled the AUC for tacrolimus. ${ }^{40,59}$ Given these findings, the manufacturer of voriconazole recommends reducing the daily dose of tacrolimus to $33 \%$ of the initial dose (i.e., 66\% reduction) when it is used in combination. ${ }^{40}$ However, in a clinical study of liver transplant recipients, trough concentrations of tacrolimus during concomitant oral administration of voriconazole $200 \mathrm{mg}$ twice/day increased nearly 10 -fold in the first subject, whose condition had been previously stabilized with tacrolimus $2 \mathrm{mg}$ /day.

The investigators had previously performed an in vitro study in human liver microsomes in which the concentration of voriconazole required to inhibit tacrolimus metabolism by $50 \%$ was $10.4 \pm 4.3 \mu \mathrm{g} / \mathrm{ml}$. The inhibition was concentration dependent. At concentrations of voriconazole 5-, 10-, and 50-fold higher than 
those of tacrolimus, tacrolimus metabolism was inhibited by $20 \%, 53 \%$, and $76 \%$, respectively. Because typical maximum and trough serum concentrations of voriconazole clinically achieved after oral dosages of $200 \mathrm{mg}$ twice/day are $2.12-4.8$ and $1.4-1.78 \mu \mathrm{g} / \mathrm{ml}$, respectively, the investigators expected an in vivo interaction of $50 \%$ or less. The magnitude of the in vivo interaction appeared to be greater than that predicted by using the in vitro data. They hypothesized that high voriconazole concentrations in the gastrointestinal tract inhibited intestinal CYP3A4 metabolism of tacrolimus, similar to the interaction observed with ketoconazole and tacrolimus. ${ }^{23}$

\section{Posaconazole}

Tacrolimus AUCs increased 4.5-fold with the addition of oral posaconazole $400 \mathrm{mg}$ twice/day for 8 days. ${ }^{24}$ Of note, the interaction of posaconazole with tacrolimus was greater than that observed with cyclosporine, perhaps because a relatively high dose of posaconazole was used. ${ }^{25}$

\section{Drug Interactions Between Azoles and Sirolimus}

\section{Ketoconazole}

In healthy subjects, the addition of oral ketoconazole $200 \mathrm{mg} /$ day for 10 days to oral sirolimus $5 \mathrm{mg} /$ day decreased the oral clearance of sirolimus by $90 \%$, similar to its effect on tacrolimus. ${ }^{60}$ These findings were confirmed in a prospective study in six kidney transplant recipients. ${ }^{61}$ However, careful monitoring and dosage adjustment allowed investigators to maintain appropriate plasma concentrations of sirolimus.

\section{Fluconazole}

To our knowledge, no randomized studies have been performed to assess the interaction between fluconazole and sirolimus, and interactions have largely been implied from observations between azoles and other CYP3A4 or P-gp substrate drugs, such as cyclosporine and tacrolimus. However, the management of drug interactions involving sirolimus may be complicated, even during the coadministration of weak CYP3A4 inhibitors such as fluconazole. One report describes a kidney transplant recipient who began sirolimus therapy on day 5 after transplantation and oral fluconazole $200 \mathrm{mg}$ /day on day 25 after transplantation. ${ }^{62}$ Despite a preemptive reduction in the dosage of sirolimus from 4 to $3 \mathrm{mg} /$ day on day 26 and an additional reduction to $2 \mathrm{mg} /$ day on day 30 , the trough concentration of sirolimus almost doubled by day 29 and tripled by day 32 .

\section{Voriconazole}

The effect of voriconazole on the pharmacokinetics of sirolimus is even more pronounced than its effect on tacrolimus. In a single-blinded, randomized, placebo-controlled, two-period crossover study of healthy subjects, oral voriconazole $400 \mathrm{mg}$ twice/day for day 1 followed by $200 \mathrm{mg}$ twice/day for 8 days increased the AUC of sirolimus (administered as a 2-mg single oral dose on day 4) by a mean of 11 -fold. ${ }^{40}$ Based on these findings, the coadministration of voriconazole and sirolimus is contraindicated. However, in a recent case series in eight patients whose sirolimus dose was initially reduced by $90 \%$, concomitant administration of voriconazole and sirolimus for a median of 33 days (range 3-100 days) resulted in trough sirolimus levels similar to those obtained before the administration of voriconazole. ${ }^{63}$ No obvious, clinically significant toxicity from either drug was observed. Serious adverse events were observed in two patients in whom sirolimus dosage was not adjusted during voriconazole administration.

\section{Other Azoles}

To date, no data are available regarding drugdrug interactions between itraconazole or posaconazole and sirolimus.

\section{Effect of Intravenous versus Oral Administration on Drug Interactions Between Immunosuppressants and Azoles}

Because CYP3A4 is expressed in the gastrointestinal tract wall and in the liver, inhibition of CYP3A 4 by azoles can occur at both sites. Therefore, inhibition of CYP3A-mediated metabolism of immunosuppressants is more pronounced when the immunosuppressant is administered orally than when it is given intravenously. Dual cooperativity of CYP enzymes and P-gp in the gastrointestinal tract contributes to remarkable differences in the oral bioavailability of immunosuppressants observed with ketoconazole versus fluconazole. Because immunosuppressants are substrates of P-gp and because ketoconazole (but not fluconazole) 
inhibits P-gp, ketoconazole inhibition of Pgp-mediated immunosuppressant efflux increases the oral bioavailability of cyclosporine more than fluconazole. For example, the administration of oral ketoconazole $200 \mathrm{mg}$ /day to five healthy subjects increased the oral and hepatic bioavailabilities of cyclosporine 2.6- and 1.2-fold, respectively. ${ }^{64}$ Intravenous administration of fluconazole $400 \mathrm{mg} /$ day, a less potent CYP3A4 inhibitor than ketoconazole, reduced the clearance of intravenously administered cyclosporine by only $21 \%{ }^{65}$ Similar effects were observed with oral tacrolimus. ${ }^{65-68}$ The administration of oral ketoconazole doubled the oral bioavailability of tacrolimus, whereas the addition of intravenous fluconazole reduced tacrolimus clearance by only $16-20 \%$.

\section{Recommendations for the Clinical Management of Drug Interactions Between Immunosuppressants and Azoles}

Transplant recipients generally receive numerous drugs, many of which induce, inhibit, or are metabolized by CYP3A4 or utilize the P-gP transport system. Therefore, assessment and management of drug interactions in this population are complex, and the application of generalized recommendations or guidelines in these patients is difficult. Reliable data are lacking, and clinicians should use caution when applying recommendations.

First, the practice of using in vitro and in vivo scaling procedures to predict in vivo effects of coadministered CYP inhibitors on drug interactions is limited. Although in vitro studies provide useful screening tools and a rationale for in vivo human investigations, human data should be used whenever possible. ${ }^{17}$

Second, dosages of immunosuppressants may need to be adjusted. Table 2 provides recommended dosage reductions of immunosuppressants during concomitant therapy with immunosuppressants and azoles. ${ }^{24,25,40,69-71}$ Although the purpose of Table 2 is to suggest dosage adjustments, interpatient variability can substantially affect the occurrence, magnitude, and clinical significance of these drug interactions.

Third, although enzyme inhibition is observed immediately after the first dose of an inhibitor is administered, minimal data are available regarding the duration of inhibition after its discontinuation. The half-life of the inhibitor and its protein binding can affect the duration of inhibition. In general, after an azole is discon-
Table 2. Recommended Percentage Dose Reductions of Immunosuppressants During Concomitant Azole Therapy ${ }^{24,25,40,69-71}$

\begin{tabular}{lccc}
\hline Azole & Cyclosporine & Tacrolimus & Sirolimus \\
\hline Ketoconazole & $70-80$ & $50-60$ & $80-90$ \\
$\begin{array}{l}\text { Fluconazole } \\
(\geq 200 \mathrm{mg} / \text { day })\end{array}$ & $21-50^{\mathrm{a}}$ & $40^{\mathrm{b}}$ & $50-70^{\mathrm{c}}$ \\
$\begin{array}{l}\text { Itraconazole } \\
\text { Voriconazole }\end{array}$ & $50-60$ & $50-60$ & No data \\
Posaconazole & $0-30^{\mathrm{f}}$ & $66^{\mathrm{d}}$ & $90^{\mathrm{e}}$ \\
\hline
\end{tabular}

${ }^{a}$ Extent of interaction depends on the route of administration of cyclosporine (see text).

bBased on studies of low-dose fluconazole $100 \mathrm{mg} /$ day.

'Based on limited data (see text).

${ }^{\mathrm{d}}$ Variable (see text).

${ }^{\mathrm{e}}$ Used in clinical practice. Coadministration is contraindicated according to the manufacturer (see text).

Based on limited data (see text).

${ }^{\mathrm{g}}$ At the time of writing.

tinued, a mean of 7-10 days is required for concentrations of immunosuppressants to return to baseline values.

Fourth, plasma concentrations of immunosuppressants should be monitored at the start of therapy, throughout combined use, and, most important, after concomitant azole therapy is discontinued.

Fifth, recommendations in the literature suggest preemptive dosage reductions of immunosuppressants when an azole is added. However, most lack validity. Therefore, they are not the standard of practice among transplant clinicians who are concerned about an increased risk of rejection in patients prescribed preemptive dosage reductions of immunosuppressants during concomitant therapy with azoles. Additional studies are needed to assess the validity and clinical outcomes of preemptive dosage reduction.

\section{Additional Factors Affecting Drug Interactions}

Both drug- and patient-related factors help determine the susceptibility to potential drug interactions. ${ }^{28}$ Factors such as genomic variability, sex, ethnicity, and disease states that alter plasma drug concentrations can affect the magnitude and clinical significance of drug interactions. ${ }^{17}$ The effect of wide interpatient variability in the absorption, distribution, metabolism, and clearance of immunosuppressants on the magnitude and clinical significance of drug interactions cannot be overemphasized. . $^{28,47}$

Sex-related differences can influence the 
magnitude of drug interactions between azoles and immunosuppressants. ${ }^{28}$ Tacrolimus pharmacokinetics were compared in 11 male and eight female kidney transplant recipients with and without the concomitant use of ketoconazole. The coadministration of oral ketoconazole and intravenous tacrolimus decreased tacrolimus clearance significantly more in the female patients than in the male patients. When both drugs were administered orally, a significantly greater increase in absolute bioavailability was observed in the female subjects. ${ }^{68}$ Researchers theorize that these sexrelated differences could result from lower metabolic capacity of the intestinal microsomes in male subjects than in premenopausal female subjects or from high P-gp activity in female subjects that increases the efficiency of CYPmediated metabolism. ${ }^{28}$

Ethnicity affects cyclosporine pharmacokinetics because hepatic CYP3A4 activity is increased in some groups. ${ }^{28}$ The oral bioavailability of tacrolimus was significantly lower in African-American healthy volunteers and kidney transplant recipients than other subjects perhaps because of differences in intestinal P-gp and CYP3A4 metabolism rather than differences in hepatic metabolism. ${ }^{28,72}$ Differences in the oral bioavailability of tacrolimus result in differences in the dose response. Among kidney transplant recipients, African-Americans require daily doses of tacrolimus 37\% higher than those needed by Caucasians to achieve similar plasma concentrations. ${ }^{73}$

Finally, in addition to factors just discussed, inflammatory small-bowel disease, cirrhosis, stress, infections, poor nutritional status, and increased age decrease the amount and activity of CYP3A4 present in tissues.

\section{Advantages of Drug Interactions}

Although drug-drug interactions are usually considered undesirable events, they can be beneficial. Therapy with immunosuppressants can be cost-prohibitive. Reduction of daily doses of immunosuppressants due to ketoconazoleinduced inhibition of their clearance can reduce therapeutic doses of the immunosuppressants by $70-85 \%$. This drug combination has proved to be well tolerated and effective, and it is considered less costly than cyclosporine monotherapy. ${ }^{74}$

Investigators who reviewed the clinical and cost-saving potential of cyclosporine drug interactions concluded that ketoconazole appeared to be the best candidate for reducing the financial burden of long-term immunosuppressive therapy without sacrificing patients' well-being. ${ }^{74}$ A 5-year study of patients receiving combined ketoconazole and cyclosporine showed no clinical difference in outcomes, including renal function, hepatic function, blood pressure, use of antihypertensive drugs, or patient or graft survival. ${ }^{73}$ The combined use of ketoconazole and cyclosporine could decrease the yearly perpatient cost of cyclosporine by approximately $\$ 3750$, which could translate to a national annual savings of more than $\$ 100$ million in patients undergoing solid organ transplantation. Other reported advantages were decreased frequencies of infections and episodes of acute rejection in patients who received ketoconazole and cyclosporine versus placebo and cyclosporine. ${ }^{74}$

A 10-year follow-up study of transplant patients receiving cyclosporine alone or combined with ketoconazole confirmed the continuity of cyclosporine dosage reduction and cost savings. ${ }^{75}$ Clinical benefits associated with combination therapy included similar frequencies of acute-rejection episodes in both groups, although the frequency and rates of unfavorable responses to therapy were higher in the control group. The frequency of chronic allograft nephropathy was also significantly lower in the ketoconazole group than in the control group; this difference was sustained after 10 years. However, data have suggested that the use of ketoconazole to reduce the dosage of cyclosporine may compromise long-term graft survival and increase the risk of late acute or chronic rejection. A potentially detrimental effect of ketoconazole on graft survival might occur because of the delayed and decreased maximum concentrations and because of the flattened or unpredictable absorption of cyclosporine observed during concomitant therapy. ${ }^{76}$ In the absence of definitive answers and given the conflicting information, the benefits of combination therapy in terms of infections and graft survival must be investigated further.

Few clinical trials have been conducted to evaluate the economic or clinical outcomes associated with the combined use of fluconazole and cyclosporine. A 3-month course of cyclosporine combined with fluconazole versus clotrimazole resulted in an estimated drug savings of $\$ 900 /$ patient, with a significantly decreased rate of fungal infections in the group receiving fluconazole. ${ }^{77}$

Itraconazole coadministration with cyclosporine 
decreased the mean dose requirement for cyclosporine by $50 \%$ and resulted in an mean cost savings of $\$ 233 /$ patient/month. ${ }^{74}$ Similar cost savings were reported with the combined use of ketoconazole and sirolimus. ${ }^{61}$ The cost of sirolimus $2 \mathrm{mg} /$ day was $\$ 625 / \mathrm{month}$ compared with $\$ 86$ for the combination of sirolimus 0.25 $\mathrm{mg}$ /day and ketoconazole $200 \mathrm{mg} /$ day.

The cost of tacrolimus was also reduced by $57 \%$ when tacrolimus was used concomitantly with ketoconazole. Additional benefits included a decrease in the total days of hospitalization, fungal skin infections, and rate of acute rejection, and a substantial improvement in graft function. ${ }^{78}$

Reports suggest potential economic benefits of fluconazole or itraconazole combined with immunosuppressants. However, ketoconazole remains the drug of choice for this indication because it is the most extensively studied azole and the least expensive and most potent inhibitor. Because only low doses of ketoconazole are needed to achieve major reductions in dosages of immunosuppressants, fewer adverse effects and similar or improved economic benefits can be achieved with ketoconazole than with other azoles.

\section{Drug Interactions Between Azoles and Investigational Immunosuppressants}

Everolimus is an investigational immunosuppressant that is structurally similar to sirolimus and that is intended to be given in combination with cyclosporine after solid organ transplantation. Because everolimus is a substrate of P-gp and CYP3A4, CYP3A5, and CYP2C8, a number of drug-drug interactions with azoles can occur. Although the administration of fluconazole has no substantial effect on the pharmacokinetics of everolimus, administration of itraconazole decreases its clearance by $74 \%{ }^{79}$

In subjects administered oral ketoconazole 200 $\mathrm{mg}$ twice/day for 8 days, a single 1-mg dose of everolimus coadministered with ketoconazole on day 4 increased the AUC 15-fold. The interaction was evident 24 hours after the administration of everolimus, and plasma concentrations of everolimus were detectable until day 24. The authors concluded that the concomitant use of ketoconazole and everolimus should be avoided if possible. ${ }^{80}$ This result emphasizes the need to monitor drug concentrations and to further evaluate drug interactions with everolimus.

\section{Conclusion}

Drug interactions between azoles and immunosuppressants are agent specific and depend on the potency of the azole inhibition of CYP and P-gp, the plasma concentrations of each agent, the drug formulation, and interpatient variability. Trough concentrations of cyclosporine, tacrolimus, and sirolimus must be monitored, and their dosages must be adjusted accordingly when an azole is added or discontinued. Clinicians should monitor patients for toxicity and loss of efficacy a few days to a week after starting and 7-10 days after discontinuing combination therapy.

\section{Acknowledgment}

Many thanks to Jamie Park, Pharm.D., for her careful review and thoughtful suggestions regarding the manuscript.

\section{References}

1. McNeil MM, Nash SL, Hajjeh RA, et al. Trends in mortality due to invasive mycotic diseases in the United States, 1980-1997. Clin Infect Dis 2001;33:641-7.

2. Kontoyiannis DP, Mantadakis E, Samonis G. Systemic mycoses in the immunocompromised host: an update in antifungal therapy. J Hosp Infect 2003;53:243-58.

3. Rubin RH. Overview: pathogenesis of fungal infections in the organ transplant recipient. Transpl Infect Dis 2002;4(suppl 3):12-17.

4. Lin SJ, Schranz J, Teutsch SM. Aspergillosis case-fatality rate: systematic review of the literature. Clin Infect Dis 2001;32(3):358-66.

5. Fung JJ. Fungal infection in liver transplantation. Transpl Infect Dis 2002;4(suppl 3):18-23.

6. Fishman JA. Overview: fungal infections in the transplant patient. Transpl Infect Dis 2002;4(suppl 3):3-11.

7. Singh N. Treatment of opportunistic mycoses: how long is long enough? Lancet Infect Dis 2003;3:703-8.

8. Rubin RH, Ikonen T, Gummert JF, Morris RE. The therapeutic prescription for the organ transplant recipient: the linkage of immunosuppression and antimicrobial strategies. Transpl Infect Dis 1999;1:29-39.

9. Albengres E, Le Louet H, Tillement JP. Systemic antifungal agents. Drug interactions of clinical significance. Drug Saf 1998;18:83-97.

10. Bates DW, Yu DT. Clinical impact of drug-drug interactions with systemic azole antifungals. Drugs Today (Barc) 2003;39:801-13.

11. Katz HI. Drug interactions of the newer oral antifungal agents. Br J Dermatol 1999;141(suppl 56):26-32.

12. Venkatakrishnan K, von Moltke LL, Greenblatt DJ. Effects of the antifungal agents on oxidative drug metabolism: clinical relevance. Clin Pharmacokinet 2000;38:111-80.

13. Gillum JG, Israel DS, Polk RE. Pharmacokinetic drug interactions with antimicrobial agents. Clin Pharmacokinet 1993;25:450-82.

14. Venkatakrishnan K, Von Moltke LL, Greenblatt DJ. Human drug metabolism and the cytochromes P450: application and relevance of in vitro models. J Clin Pharmacol 2001;41: 1149-79.

15. Zhang Y, Benet LZ. The gut as a barrier to drug absorption: combined role of cytochrome P450 3A and P-glycoprotein. Clin Pharmacokinet 2001;40:159-68. 
16. Davidson MH. Does differing metabolism by cytochrome P450 have clinical importance? Curr Atheroscler Rep 2000;2:14-19.

17. Dresser GK, Spence JD, Bailey DG. Pharmacokineticpharmacodynamic consequences and clinical relevance of cytochrome P450 3A4 inhibition. Clin Pharmacokinet 2000;38:41-57.

18. Hall SD, Thummel KE, Watkins PB, et al. Molecular and physical mechanisms of first-pass extraction. Drug Metab Dispos 1999;27:161-6.

19. Lin JH, Lu AY. Inhibition and induction of cytochrome P450 and the clinical implications. Clin Pharmacokinet 1998;35:361-90

20. Apseloff G, Hilligoss DM, Gardner MJ, et al. Induction of fluconazole metabolism by rifampin: in vivo study in humans. J Clin Pharmacol 1991;31:358-61.

21. Tucker WS Jr, Snell BB, Island DP, Gregg CR. Reversible adrenal insufficiency induced by ketoconazole. JAMA 1985;253:2413-14.

22. Christians U, Strom T, Zhang YL, et al. Active drug transport of immunosuppressants: new insights for pharmacokinetics and pharmacodynamics. Ther Drug Monit 2006;28(1):39-44.

23. Venkataramanan $\mathrm{R}$, Zang S, Gayowski T, Singh N. Voriconazole inhibition of the metabolism of tacrolimus in a liver transplant recipient and in human liver microsomes. Antimicrob Agents Chemother 2002;46:3091-3.

24. Sansone A, Belle D, Statkevich P, et al. Effect of posaconazole on the pharmacokinetics of tacrolimus in healthy volunteers [abstr]. In: Abstracts of the 43rd interscience conference on antimicrobial agents and chemotherapy, Chicago, IL, September 14-17, 2003. Washington, DC: American Society for Microbiology, 2003:1603.

25. Courtney RD, Statkevich P, Laughlin M, et al. Effect of posaconazole on the pharmacokinetics of cyclosporine [abstr]. In: Abstracts of the 4lst interscience conference on antimicrobial agents and chemotherapy, Chicago, IL, December 16-19, 2001. Washington, DC: American Society for Microbiology, 2001:A27.

26. Lo A, Burckart GJ. P-glycoprotein and drug therapy in organ transplantation. J Clin Pharmacol 1999;39:995-1005.

27. Benet LZ, Cummins CL, Wu CY. Transporter-enzyme interactions: implications for predicting drug-drug interactions from in vitro data. Curr Drug Metab 2003;4:393-8.

28. Christians U, Jacobsen W, Benet LZ, Lampen A. Mechanisms of clinically relevant drug interactions associated with tacrolimus. Clin Pharmacokinet 2002;41:813-51.

29. Wang EJ, Lew K, Casciano CN, Clement RP, Johnson WW. Interaction of common azole antifungals with $\mathrm{P}$ glycoprotein. Antimicrob Agents Chemother 2002;46:160-5.

30. Bertz RJ, Granneman GR. Use of in vitro and in vivo data to estimate the likelihood of metabolic pharmacokinetic interactions. Clin Pharmacokinet 1997;32:210-58.

31. Thummel KE, Wilkinson GR. In vitro and in vivo drug interactions involving human CYP3A. Annu Rev Pharmacol Toxicol 1998;38:389-430.

32. McConn DJ II, Lin YS, Allen K, Kunze KL, Thummel KE. Differences in the inhibition of cytochromes P450 3A4 and 3A5 by metabolite-inhibitor complex-forming drugs. Drug Metab Dispos 2004;32:1083-91.

33. Gibbs MA, Thummel KE, Shen DD, Kunze KL. Inhibition of cytochrome P-450 3A (CYP3A) in human intestinal and liver microsomes: comparison of $\mathrm{K}_{\mathrm{i}}$ values and impact of CYP3A5 expression. Drug Metab Dispos 1999;27:180-7.

34. Bachmann KA, Lewis JD. Predicting inhibitory drug-drug interactions and evaluating drug interaction reports using inhibition constants. Ann Pharmacother 2005;39:1064-72.

35. Neal JM, Kunze KL, Levy RH, O'Reilly RA, Trager WF. Kiiv, an in vivo parameter for predicting the magnitude of a drug interaction arising from competitive enzyme inhibition. Drug Metab Dispos 2003;31:1043-8.

36. Maertens JA. History of the development of azole derivatives. Clin Microbiol Infect 2004;10(suppl 1):1-10.

37. Halloran PF, Gourishankar S. Principles and overview of immunosuppression. In: Norman DJ, Turka LA, eds. Primer on transplantation, 2nd ed. Mt. Laurel, NJ: American Society of Transplantation, 2001:87-97.

38. Isoherranen N, Kunze KL, Allen KE, Nelson WL, Thummel KE. Role of itraconazole metabolites in CYP3A4 inhibition. Drug Metab Dispos 2004;32:1121-31.

39. Gubbins PO, Amsden JR. Drug-drug interactions of antifungal agents and implications for patient care. Expert Opin Pharmacother 2005;6:2231-43.

40. Pfizer Inc. Vfend (voriconazole) package insert. New York, NY; 2003.

41. Pearson MM, Rogers PD, Cleary JD, Chapman SW. Voriconazole: a new triazole antifungal agent. Ann Pharmacother 2003;37:420-32.

42. Ghosal A, Hapangama N, Yuan Y, et al. Identification of human UDP-glucuronosyltransferase enzyme(s) responsible for the glucuronidation of posaconazole (Noxafil). Drug Metab Dispos 2004;32:267-71.

43. Slain D, Rogers PD, Cleary JD, Chapman SW. Intravenous itraconazole. Ann Pharmacother 2001;35:720-9.

44. Omar G, Whiting PH, Hawksworth GM, Humphrey MJ, Burke MD. Ketoconazole and fluconazole inhibition of the metabolism of cyclosporin A by human liver in vitro. Ther Drug Monit 1997;19:436-45.

45. Wexler D, Courtney R, Richards W, Banfield C, Lim J, Laughlin M. Effect of posaconazole on cytochrome P450 enzymes: a randomized, open-label, two-way crossover study. Eur J Pharm Sci 2004;21:645-53.

46. Gaston RS. Maintenance immunosuppression in the renal transplant recipient: an overview. Am J Kidney Dis 2001;38:S25-35.

47. Mahalati K, Kahan BD. Clinical pharmacokinetics of sirolimus. Clin Pharmacokinet 2001;40:573-85.

48. Dunn CJ, Wagstaff AJ, Perry CM, Plosker GL, Goa KL. Cyclosporin: an updated review of the pharmacokinetic properties, clinical efficacy and tolerability of a microemulsionbased formulation (Neoral) 1 in organ transplantation. Drugs 2001;61:1957-2016.

49. Abraham MA, Thomas PP, John GT, Job V, Shankar V, Jacob CK. Efficacy and safety of low-dose ketoconazole (50 mg) to reduce the cost of cyclosporine in renal allograft recipients. Transplant Proc 2003;35:215-16.

50. Lomaestro BM, Piatek MA. Update on drug interactions with azole antifungal agents. Ann Pharmacother 1998;32:915-28.

51. Canafax DM, Graves NM, Hilligoss DM, Carleton BC, Gardner MJ, Matas AJ. Interaction between cyclosporine and fluconazole in renal allograft recipients. Transplantation 1991;51(5):1014-18.

52. Romero AJ, Pogamp PL, Nilsson LG, Wood N. Effect of voriconazole on the pharmacokinetics of cyclosporine in renal transplant patients. Clin Pharmacol Ther 2002;71:226-34.

53. Moreno M, Latorre A, Manzanares C, et al. Clinical management of tacrolimus drug interactions in renal transplant patients. Transplant Proc 1999;31:2252-3.

54. Toda F, Tanabe K, Ito S, et al. Tacrolimus trough level adjustment after administration of fluconazole to kidney recipients. Transplant Proc 2002;34:1733-5.

55. Hairhara Y, Makuuchi M, Kawarasaki H, et al. Effect of fluconazole on blood levels of tacrolimus [abstr]. Transplant Proc 1999;31:2767.

56. Capone D, Gentile A, Imperatore P, Palmiero G, Basile V. Effects of itraconazole on tacrolimus blood concentrations in a renal transplant recipient. Ann Pharmacother 1999;33:1124-5.

57. Billaud EM, Guillemain R, Tacco F, Chevalier P. Evidence for a pharmacokinetic interaction between itraconazole and tacrolimus in organ transplant patients. Br J Clin Pharmacol 1998;46:271-2.

58. Banerjee R, Leaver N, Lyster H, Banner NR. Coadministration of itraconazole and tacrolimus after thoracic organ transplantation. Transplant Proc 2001;33:1600-2.

59. Wood N, Tan KKC, Allan R, Fielding A, Nichols DJ. Effect of voriconazole on the pharmacokinetics of tacrolimus [abstr]. In: Abstracts of the 4lst interscience conference on antimicrobial agents and chemotherapy, Chicago, IL, December 16-19, 2001. 
Washington, DC: American Society for Microbiology, 2001:A20.

60. Zimmeran JJ, Gurr JA, DiLea C. Study of the potential pharmacokinetic interaction between sirolimus (Rapamune) and ketoconazole: final report (protocol 0468E1-136-US), vol 163/2. Wyeth-Ayerst GMR-31057, 1998.

61. Thomas PP, Manivannan J, John GT, Jacob CK. Sirolimus and ketoconazole co-prescription in renal transplant recipients. Transplantation 2004;77:474-5.

62. Cervelli MJ. Fluconazole-sirolimus drug interaction. Transplantation 2002;74:1477-8.

63. Marty FM, Lowry CM, Cutler CS, et al. Voriconazole and sirolimus coadministration after allogeneic hematopoietic stem cell transplantation. Biol Blood Marrow Transplant 2006;12(5):552-9.

64. Gomez DY, Wacher VJ, Tomlanovich SJ, Hebert MF, Benet LZ. The effects of ketoconazole on the intestinal metabolism and bioavailability of cyclosporine. Clin Pharmacol Ther 1995;58:15-19.

65. Osowski CL, Dix SP, Lin LS, Mullins RE, Geller RB, Wingard JR. Evaluation of the drug interaction between intravenous high-dose fluconazole and cyclosporine or tacrolimus in bone marrow transplant patients. Transplantation 1996;61:1268-72.

66. Floren LC, Bekersky I, Benet LZ, et al. Tacrolimus oral bioavailability doubles with coadministration of ketoconazole. Clin Pharmacol Ther 1997;62:41-9.

67. Manez R, Martin M, Raman V, et al. Fluconazole therapy in transplant recipients receiving FK 506. Transplantation 1994;57:1521-3.

68. Tuteja S, Alloway RR, Johnson JA, Gaber AO. The effect of gut metabolism on tacrolimus bioavailability in renal transplant recipients. Transplantation 2001;71:1303-7.

69. Taro Pharmaceuticals. Ketoconazole package insert. Hawthorne, NY; 1999

70. Pfizer Roerig. Diflucan (fluconazole) package insert. New York, NY; 2004.

71. Janssen Pharmaceutica Products. Sporanox (itraconazole) package insert. Titusville, NJ; 2002.

72. Fitzsimmons WE, Bekersky I, Dressler D, Raye K, Hodosh E, Mekki Q. Demographic considerations in tacrolimus pharmacokinetics. Transplant Proc 1998;30:1359-64.

73. First MR, Schroeder TJ, Daoud J, et al. Ketoconazole is not associated with increased inpatient or outpatient resources while decreasing cyclosporine annual costs by approximately $\$ 3,300$ per patient. Presented at the 16 th annual meeting of the American Society of Transplant Physicians, Dallas, TX, May 11-14, 1997.

74. Martin JE, Daoud AJ, Schroeder TJ, First MR. The clinical and economic potential of cyclosporin drug interactions. Pharmacoeconomics 1999;15:317-37.

75. El-Agroudy AE, Sobh MA, Hamdy AF, Ghoneim MA. A prospective, randomized study of coadministration of ketoconazole and cyclosporine in kidney transplant recipients: ten-year follow-up. Transplantation 2004;77:1371-6.

76. Dominguez J, Kompatzki A, Foradori A, Norambuena R. Ketoconazole alters cyclosporine pharmacokinetic profile and may predispose to acute rejection. Transplant Proc 2003;35:2522-3.

77. Rossi SJ, Martin JE, Gelhot A, et al. A randomized prospective trial of fluconazole vs. clotrimazole for fungal prophylaxis in liver transplant recipients. Presented at the 14th annual meeting of the American Society of Transplant Physicians, Chicago, IL, May 14-15, 1995.

78. El-Dahshan KF, Bakr MA, Donia AF, Badr Ael-S, Sobh MA. Coadministration of ketoconazole to tacrolimus-treated kidney transplant recipients: a prospective randomized study. Nephrol Dial Transplant 2004;19:1613-17.

79. Kirchner GI, Meier-Wiedenbach I, Manns MP. Clinical pharmacokinetics of everolimus. Clin Pharmacokinet 2004:43:83-95.

80. Kovarik JM, Beyer D, Bizot MN, Jiang Q, Shenouda M, Schmouder RL. Blood concentrations of everolimus are markedly increased by ketoconazole. J Clin Pharmacol 2005;45:514-18. 